\title{
Obtención de ácidos grasos a partir de biomasa microalgal cultivada bajo diferentes condiciones de iluminación
}

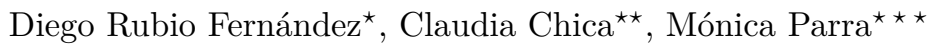 \\ Universidad de América. Bogotá - Colombia
}

FeCha DE ENTREGA: 2 DE OCTUBRE DE 2012 FECHA DE APROBACIÓN: 3 DE ABRIL DE 2013

\begin{abstract}
Resumen Se considera que los ácidos grasos provenientes de la biomasa microalgal tienen el potencial de ser utilizados como materia prima para la producción de biocombustibles como el biodiesel o para suplementos alimenticios. El tratamiento experimental consistió en cultivar las microalgas bajo diferentes parámetros de luz, incluidos el fotoperiodo y la longitud de onda. Para cada tratamiento, se cultivó la microalga Chlorella sp desde $0.5 \mathrm{ml}$ y se escaló hasta 5 litros; se determinó la velocidad de crecimiento de cada microalga, y la composición de ácidos grasos de uno de los tratamientos fue establecida.

El tratamiento de luz roja bajo un fotoperiodo de doce horas luz y doce horas oscuridad presentó la mayor tasa de crecimiento de $2.33^{9}$ células/día; los ácidos grasos obtenidos mostraron una importante cantidad de ácido oleico, indicando un fuerte potencial de la biomasa de las microalgas para ser utilizadas en la industria alimenticia.
\end{abstract}

\begin{abstract}
It is considered that microalgae fatty acids have the potential to be used as raw material for the production of biofuels such as biodiesel or food supplements. The experimental treatment consisted in the exposure of the microalgae to different light color and photoperiod. For each treatment, microalgae Chlorella sp was cultivated from a $0.5 \mathrm{ml}$ inoculum and escalated to 5 liters; the growth speed of the microalgae was determined, and the composition of fatty acids of one of the treatments was established.

The treatment of red light under a photoperiod of twelve hours of light and twelve of dark showed the highest growth rate of $2.33^{9}$ cells/day; the fatty acids obtained showed an important quantity of oleic acid, indicating
\end{abstract}

* Docente Investigador Universidad de América. Grupo de Investigación en Energías Alternativas, línea de investigación en biohidrógeno y biocombustibles. diego.rubio@profesores .uamerica.edu.co

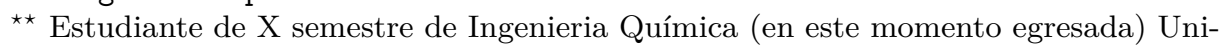
versidad de América Grupo de Investigación en Energías Alternativas, línea de investigación en biohidrógeno y biocombustibles. claurisr@hotmail.com

*** Estudiante de X semestre de Ingenieria Química (en este momento egresada) Universidad de América Grupo de Investigación en Energías Alternativas, línea de investigación en biohidrógeno y biocombustibles. monis_f1@hotmail.com 
a strong potential of the microalgae biomass for its use in alimentary industry.

Palabras Clave: ácidos grasos, biomasa, Chlorella sp, ácido oleico.

Keywords: fatty acids, biomass, Chlorella sp, oleic acid.

\section{Introducción}

Las microalgas son un grupo altamente diverso de organismos unicelulares entre los que se encuentran protistas eucaríóticos y cianobacterias procarióticas, que pueden crecer rápidamente debido a su estructura simple y tienen un alto potencial para la producción de sustancias de interés [9].

Las materias primas para la producción de lípidos y aceites son de interés prioritario, pues la demanda de este tipo de sustancias se encuentra en aumento para la producción de alimentos, la síntesis de biocombustibles o sustancias farmacéuticas de interés; los procesos de producción masiva de microalgas tienen el potencial de ser aplicados a la industria alimenticia, farmacéutica o de biocombustibles; sin embargo, el biodiesel a partir de aceites de microalgas, aun no compite en términos de costos con los aceites obtenidos de otros cultivos [1], aunque en términos de rendimiento, los cultivos de microalgas sean comparativamente superiores [2]; el conocimiento referente a los parámetros de producción de biomasa microalgal desde la escala experimental, pueden ser probados en mayores escalas, constituyéndose en tema de investigación del área de ingeniería química.

Los estudios recientes de producción de ácidos grasos a partir de microalgas, buscan principalmente determinar métodos adecuados para que los rendimientos permitan por ejemplo, producir biodiesel de forma sostenida; sin embargo, los aceites microalgales tienen otras aplicaciones importantes, entre las cuales se encuentra su uso como aditivo nutricional en alimentos para recién nacidos [12]. Los ácidos grasos poliinsaturados a partir de microalgas, tienen ventajas sobre los ácidos grasos de origen animal, principalmente de pescado, los cuales en muchos casos tienen olores desagradables, contaminación con metales pesados, presencia de colesterol, y producción variable, además de tener una composición muy heterogénea que dificulta la purificación [8].

Teniendo en cuenta la relación entre generación de biomasa y producción de lípidos, las microalgas pueden dividirse en dos grupos: de rápido crecimiento y baja acumulación de lípidos; por ejemplo, Chlorella vulgaris y de alta acumulación de lípidos pero tiempos de duplicación poblacional largos, o producción de biomasa poco eficiente, por ejemplo, Botriococcus brauni [3].

En [4] analizaron la producción de lípidos en Chlorella vulgaris y determinaron una productividad de $147 \mathrm{mg} / \mathrm{l} /$ día de ácidos grasos, concluyendo que el acoplar procesos de tipo ambiental como el tratamiento de aguas con la generación de biomasa, se pueden disminuir los costos y aumentar las eficiencias. En 6], establecieron un método para aumentar la producción de lípidos en Chlorella vulgaris, variando y ajustando las condiciones de los cultivos, concluyendo que las condiciones de cultivo influencian en la producción de biomasa y acumulación 
de ácidos grasos; en este contexto, se estableció un proceso para la producción de ácidos grasos a partir de cultivos de la microalga Chlorella sp, en los que se evaluaron la longitud de onda y el fotoperiodo como parámetros para mejorar el crecimiento y la producción de biomasa de las microalgas.

\section{Materiales y métodos}

\subsection{Microalga y condiciones de cultivo}

Se cultivó la microalga Chlorella sp obtenida en el Laboratorio de Biotecnología Vegetal de la Universidad de Antioquia. El medio de cultivo empleado fue Bold Basal BBM, (Figura 1) a temperatura constante de $25^{\circ} \mathrm{C}$.

\begin{tabular}{|c|c|c|}
\hline & Macronutrientes & $\begin{array}{c}\text { Cantidad usada para } \\
250 \mathrm{ml} \\
\end{array}$ \\
\hline & $\mathrm{NaNO}_{3}$ & $6,25 \mathrm{~g}$ \\
\hline & $\mathrm{MgSO}_{4} 7 \mathrm{H}_{2} \mathrm{O}$ & $1,88 \mathrm{~g}$ \\
\hline & $\mathrm{NaCl}$ & $0,63 \mathrm{~g}$ \\
\hline & $\mathrm{K}_{2} \mathrm{HPO}_{4}$ & $1,88 \mathrm{~g}$ \\
\hline & $\mathrm{KH}_{2} \mathrm{PO}_{4}$ & $4,38 \mathrm{~g}$ \\
\hline & $\mathrm{CaCl}_{2} .2 \mathrm{H}_{2} \mathrm{O}$ & $0,63 \mathrm{~g}$ \\
\hline & Micronutrientes & $\begin{array}{l}\text { Cantidad usada para } \\
250 \mathrm{ml}\end{array}$ \\
\hline \multirow{5}{*}{$\begin{array}{l}\text { Trace Metal } \\
\text { Solutions }\end{array}$} & $\mathrm{ZnSO}_{4} .7 \mathrm{H}_{2} \mathrm{O}$ & $2,21 \mathrm{~g}$ \\
\hline & $\mathrm{MnCl}_{2} \cdot 4 \mathrm{H}_{2} \mathrm{O}$ & $0,36 \mathrm{~g}$ \\
\hline & $\mathrm{MoO}_{3}$ & $0,18 \mathrm{~g}$ \\
\hline & $\mathrm{CuSO}_{4.5} \cdot \mathrm{H}_{2} \mathrm{O}$ & $0,39 \mathrm{~g}$ \\
\hline & $\mathrm{Co}\left(\mathrm{NO}_{3}\right)_{2} \cdot 6 \mathrm{H}_{2} \mathrm{O}$ & $0,12 \mathrm{~g}$ \\
\hline Boron Solution & $\mathrm{H}_{3} \mathrm{BO}_{3}$ & $2,86 \mathrm{~g}$ \\
\hline \multirow{2}{*}{$\begin{array}{c}\text { Alkaline EDTA } \\
\text { Solution }\end{array}$} & EDTA & $12,50 \mathrm{~g}$ \\
\hline & $\mathrm{KOH}$ & $7,75 \mathrm{~g}$ \\
\hline \multirow{2}{*}{$\begin{array}{l}\text { Acidified Iron } \\
\text { Solution }\end{array}$} & $\mathrm{FeSO}_{4.7 \mathrm{H}_{2} \mathrm{O}}$ & $1,25 \mathrm{~g}$ \\
\hline & $\mathrm{H}_{2} \mathrm{SO}_{4}$ (Conc) & $0,25 \mathrm{ml}$ \\
\hline
\end{tabular}

Figura 1. Concentraciones de macro y micronutrientes utilizados para el cultivo BBM.

\subsection{Producción de biomasa y tratamiento experimental}

Las condiciones de cultivo variaron de acuerdo con la longitud de onda utilizada y de acuerdo con el fotoperiodo (Figura 2). El fotoperiodo comprende la relación entre horas luz y horas oscuridad a las cuales se exponen los cultivos; tres fotoperiodos se establecieron, cuyas proporciones luz: oscuridad fueron, 12:12; 15:09; 18:06. La adecuación de los fotoperiodos se dio por medio de un temporizador unido a las fuentes de luz que permitió establecer diferentes periodos de luz y oscuridad. 
La longitud de onda se relaciona con el color de la luz a la cual se expusieron los cultivos; las cuatro longitudes de onda establecidas fueron roja, azul, verde y amarilla, y fueron aplicadas a los cultivos microalgales por medio de recipientes adaptados para aislar los cultivos y permitir el contacto de estos solo con la respectiva longitud de onda según el tratamiento experimental; las longitudes de onda fueron aplicadas desde los volúmenes de cultivo de $500 \mathrm{ml}$ hasta los volúmenes de $5 \mathrm{~L}$.

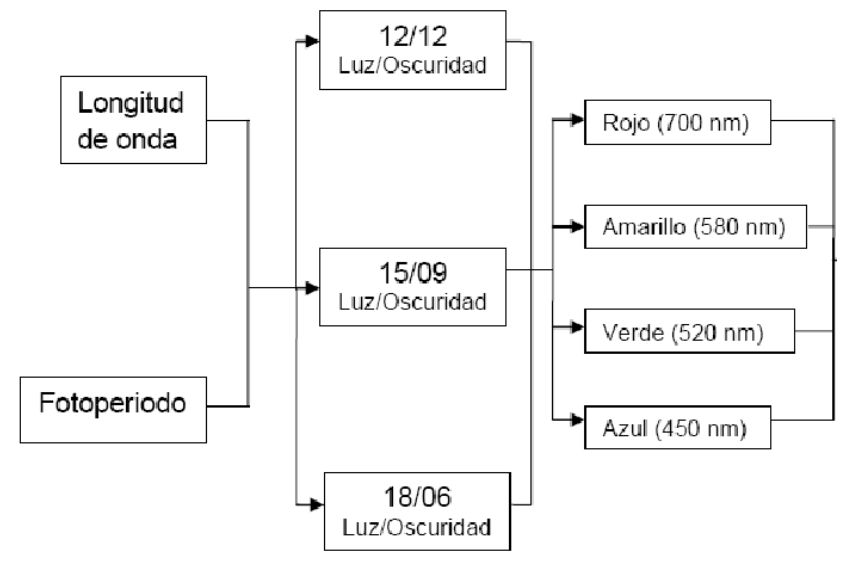

Figura 2. Condiciones experimentales planteadas para la producción de biomasa, fotoperiodo y longitud de onda. Fuente: Los Autores.

Se realizaron 12 ensayos en los que se comparó la producción de biomasa microalgal expuestas a condiciones de longitud de onda y fotoperiodo diferentes. Los cultivos se realizaron en recipientes transparentes de vidrio, a los cuales se les implemento un sistema para agitación y monitoreo de la temperatura. Se llevo a cabo un proceso de escalamiento desde $10 \mathrm{ml}$ hasta 5 litros. Las microalgas fueron cultivadas de forma mixotrófica, suministrando $\mathrm{CO}_{2}$ a partir del medio de cultivo (EDTA). El aire para agitación fue provisto a los cultivos a través de una bomba marca Hermle E101 con capacidad de diez y nueve litros de bombeo. El monitoreo de la temperatura se estableció por medio de un controlador digital de temperatura, este parámetro se controlo por medio de una termocupla que permitió mantener la temperatura entre los $25^{\circ} \mathrm{C}$ y los $27^{\circ} \mathrm{C}$.

\subsection{Determinación de los patrones de crecimiento de los cultivos}

El seguimiento a los cultivos microalgales se llevó a cabo por conteos celulares diarios en cámara de Neubauer. La tasa de crecimiento poblacional se determinó por la ecuación 1 


$$
\mu_{i}=\frac{\Delta \text { Número de células }}{\Delta \text { Tiempo }}
$$

Donde $\mu$ representa la tasa de crecimiento poblacional obtenida según el fotoperiodo y la longitud de onda trabajados.

\subsection{Tratamiento de la biomasa}

La biomasa microalgal fue separada del medio de cultivo por centrifugación y posteriormente se estableció un proceso de extracción de ácidos grasos por el método "Blight and Dyer" [1] para una de las muestras obtenidas.

\subsection{Cuantificación de lípidos y perfil de ácidos grasos}

La determinación de ácidos grasos fue realizada por cromatografía de gases, a la biomasa obtenida bajo luz roja. Los patrones de ácidos grasos utilizados fueron ácido palmítico (C16:0); ácido palmitoleico (C16:1); ácido heptadecanoico (C17:0); ácido esteárico (C18:0); ácido oléico (C18:1n-9c, C18:1n-7); ácido linolelaídico (C18:2n-6t); ácido linoléico (C18:2n-6t); ácido araquídico (C20:0); ácido cis_11eicosenoico (C20:1n-9); ácido $\alpha$-linoléico; ácido behénico (C22:0).

\section{Resultados}

\subsection{Crecimiento microalgal bajo diferentes longitudes de onda}

La mayor producción de biomasa se dio para el fotoperiodo 12:12 (Figura 3A), con una concentración de células por mililitro superior al fotoperiodo 18:6 (Figura 3B) $\mathrm{y}$ al fotoperiodo 15:9 (Figura $3 \mathrm{C}$ ). Bajo la longitud de onda roja se presentaron mayores crecimientos de la biomasa en los cultivos establecidos para los tres fotoperiodos (Figura 3A, 3B y $3 \mathrm{C}$ ).

Las tasas de crecimiento poblacional reflejan que la longitud de onda roja fue la que permitió obtener los valores más altos en el fotoperiodo 12:12, 18:06 y 15:09 (Figura 4).

\subsection{Obtención de lípidos de la biomasa microalgal}

Las concentraciones de ácidos grasos para la biomasa obtenida en fotoperiodo 12:12 y longitud de onda roja, presenta altos niveles de ácido palmítico, ácido oleico y ácido linoleico (Cuadro 1). El porcentaje de ácidos grasos poliinsaturados fue inferior al de ácidos grasos monosaturados y saturados, siendo estos últimos los que presentaron mayor porcentaje (Cuadro 2). 

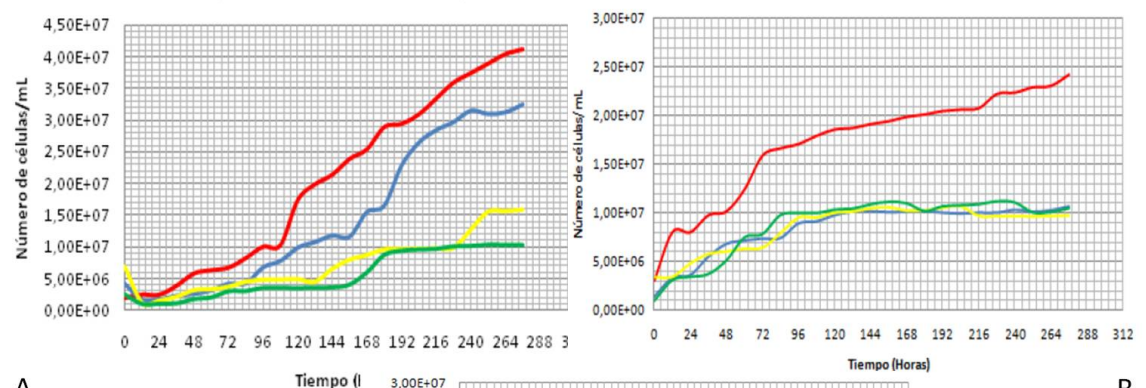

A

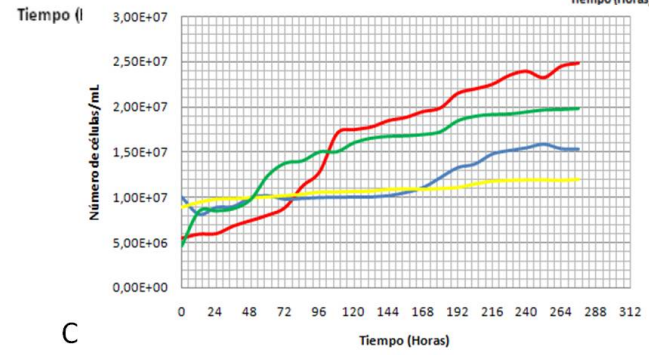

Figura 3. Patrones de crecimiento de cultivos de Chlorella sp bajo las condiciones experimentales fotoperiodo en horas y longitud de onda. $3 \mathrm{~A}$ : Fotoperiodo 12:12; $3 \mathrm{~B}$ : Fotoperiodo 18:06; 3C: Fotoperiodo 15:09. Los colores de las gráficas corresponden a las longitudes de onda bajo las cuales se llevaron a cabo los cultivos. Fuente: Los Autores.

\begin{tabular}{|c|c|c|c|}
\hline $\begin{array}{c}\text { Longitud } \\
\text { de onda }\end{array}$ & $\begin{array}{c}\text { Fotoperiodo } \\
12 / 12\end{array}$ & $\begin{array}{c}\text { Fotoperiodo } \\
18 / 06\end{array}$ & $\begin{array}{c}\text { Fotoperiodo } \\
15 / 09\end{array}$ \\
\hline Rojo & $2,33 \mathrm{E}+09$ & $2,16 \mathrm{E}+09$ & $1,64 \mathrm{E}+08$ \\
\hline Azul & $1,76 \mathrm{E}+09$ & $7,80 \mathrm{E}+08$ & $8,02 \mathrm{E}+06$ \\
\hline Amarillo & $8,48 \mathrm{E}+08$ & $9,37 \mathrm{E}+08$ & $8,96 \mathrm{E}+07$ \\
\hline Verde & $1,04 \mathrm{E}+09$ & $8,41 \mathrm{E}+08$ & $5,56 \mathrm{E}+06$ \\
\hline
\end{tabular}

Figura 4. Tasas de crecimiento poblacional de Chlorella sp determinadas según las condiciones experimentales. Fuente: Los Autores.

\section{Discusión}

El éxito de la producción de biomasa microalgal para la obtención de sustancias de interés, depende de factores que aparentemente son poco similares como la fisiología de los cultivos y la tecnología [5]. Por esta razón, aunque muchos de los factores que pueden afectar el cultivo de las microalgas se relacionan, por ejemplo, como el caso de la transferencia de calor a los cultivos microalgales y el tipo de iluminación suministrado a estos en el caso de fuentes de luz que transmiten luz y calor, o la agitación y la transferencia de masa, su análisis individual permite 


\begin{tabular}{lc}
\hline Ácido Graso & Biomasa \\
\hline Palmítico & 23,13 \\
\hline Palmitoleico & 0,40 \\
\hline Heptadecanóico & 0,26 \\
\hline Esteárico & 4,76 \\
\hline Oléico & 26,33 \\
\hline Linolelaílico & 0,39 \\
\hline Linoléico & 14,09 \\
\hline Araquídico & 6,34 \\
\hline Cis_11-Eicosenoico & 0,52 \\
\hline Alfa-linoléico & 9,03 \\
\hline Behénico & 0,24 \\
\hline
\end{tabular}

Cuadro 1. Perfil de ácidos grasos (\%) de la microalga Chlorella sp, cultivada bajo condiciones de fotoperiodo 12:12 y longitud de onda roja. Fuente: Los Autores.

\begin{tabular}{lc}
\hline Tipo de ácido graso & Biomasa \\
\hline Poliinsaturados (Pufas) & 23,51 \\
\hline Monosaturados (Mufas) & 32,92 \\
\hline Saturados (Sfas) & 34,72 \\
\hline
\end{tabular}

Cuadro 2. Porcentaje de ácidos grasos obtenidos de la muestra microalgal según su grado de saturación. Fuente: Los Autores.

establecer de una forma más directa, los valores específicos que permiten la optimización de la fisiología de un cultivo, lo cual se traduce en un aumento de la eficiencia del proceso. Por otra parte, al analizar factores conjuntos, se hace difícil establecer cuáles son los factores que benefician o perjudican un proceso relacionado con la producción de bienes a través de técnicas biotecnológicas.

Según [5], es imperativo el entendimiento de cómo las variables relacionadas con los procesos afectan la producción de biocompuestos, en el caso de la biotecnología de microalgas. La evaluación del fotoperiodo, para la producción de biomasa, indica que la proporción luz: oscuridad de 12:12 horas, fue el parámetro que mostró mayor incidencia en la producción de la biomasa, mientras que la longitud de onda roja fue la que permitió la generación de biomasa con mayor eficiencia. Sin embargo, la acumulación de algunos ácidos grasos no fue óptima, lo que concuerda con la teoría referente a la relación del crecimiento de microalgas y la acumulación de ácidos grasos. 
La producción de ácidos grasos, a nivel de microalgas es superior a otras fuentes de estos materiales como la soya o el algodón. Dentro del perfil de ácidos grasos obtenidos, se hace importante determinar cuál es el potencial de esta materia prima desde el punto de vista cualitativo, pues una cantidad alta de ácidos grasos libres limitaría el proceso de transesterificación alcalina para la producción de biodiesel [10].

La composición de ácidos grasos de microalgas, del género Chlorella, ha sido estudiada principalmente con respecto a las condiciones de cultivo; se estableció [7] que hay un perfil definido para ciertas condiciones de cultivo de 14:0, 16:0, 16:1, 16:2, 16:3,18:0,18:1,18:2,18:3; este perfil coincide en algunos ácidos grasos contenidos en las muestras analizadas, particularmente en el caso de 16:0, 18:1, 18:2; sin embargo, la presencia de ácidos grasos como 20:0, 20:1 o 20:2 se asocia con impurezas en la muestra o posiblemente contaminación. Este puede haber sido el caso de este estudio, pues los cultivos fueron monoalgales pero no completamente estériles, pues durante los procesos de escalamiento principalmente a volúmenes superiores a $5 \mathrm{~L}$ se presentó contacto con aire no estéril.

\section{Conclusión}

El proceso establecido permitió comparar diferentes tipos de luz para obtener biomasa microalgal y de esta fue posible obtener una muestra de ácidos grasos. Los resultados obtenidos indican que los cultivos de microalgas bajo luz roja $\mathrm{y}$ fotoperiodo 12:12 tienden a generar concentraciones de microalgas altas en periodos cortos; sin embargo no pudo obtenerse información referente a la cantidad y calidad de ácidos grasos bajo otras longitudes de onda. Durante el proceso se evidenció que la posibilidad de escalar este tipo de procesos para establecer producciones a nivel industrial depende de la capacidad de generar biomasa en volumenes y concentraciones elevadas, lo cual a su vez depende de un completo conocimiento de la fisiología de los cultivos de microalgas en niveles industriales, problemas muy relacionados con la transferencia de masa, calor y el diseño de condiciones adecuadas que pueden variar según las especies estudiadas.

\section{Referencias}

1. Bligh, E.G., Dyer, W,J.: A rapid method of total lipid extraction and purification. Canadian Journal of Biochemistry and Physiology 37: 911-917. (1959)

2. Chisti, J.: Biodiesel from microalgae, research review paper. Biotechnology Advances 25: 294-306 (2007)

3. Dayananda, C., Sarada, R., Usha, R.M., Shamala, T.R., Ravishankar, G.A.: Autotrophic cultivation of Botryocuccus braunii for the production of hydrocarbons and exopolysaccharide in various media. Biomass Bioenergy 31:87-93. (2007)

4. Feng, Y., Li, C., Zhang, D.: Lipid Production of Chlorella vulgaris cultured in artificial wastewater médium. Bioresource Technology 102:101-105. (2011)

5. Grobbelaar, J.U.: Physiological and Technological considerations for optimising mass algal cultures. Journal of Applied Phycology 12: 201-206. (2000) 
6. Ming, J., Cheng, L,H., Xu,X., Zhang, L., Chen,H.: Enhanced lipid production of Chlorella vulgaris by adjustment of cultivation conditions. Bioresource Technology 101:6797-6804. (2010)

7. Petkov, G., Garcia, G.: Which are Fatty Acids of the Green Algae Chlorella?. Biochemical Systematics and Ecology 35: 281-285. (2007)

8. Robles, A., Molina, E., Giménez, A., Ibañes, M.J.: Downstream processing of algal polyunsaturated fatty acids. Biotechnology Advances 16(3): 517-580 (1998)

9. Sforza, E., Bertucco, A., Morosinotto, T., Giacometti, G.M.: Vegetal oil from microalgae: species selection and optimization of growth parameters. Chemical Engineering Transactions 20: 199-204. (2010)

10. Sharma, Y.C., Singh, B.: Development of Biodiesel: Current scenario. Renewable and Sustainable Energy Reviews 13:1646-1651. (2009)

11. Sheenan, J., Dunahay, T., Benemann, T., Roessler, P.: A look back at the US department of energy's aquatic species program: biodiesel from algae. (1998)

12. Varfolomeev, S.D., Wasserman, L.A.: Microalgae as source of biofuel, food, fodder, and medicines. Applied Biochemistry and Microbiology 47(9): 789-907. (2011) 